Résumés des conférences et travaux

\title{
Hagiographie, idéologies cléricales et pratiques sociales au Moyen Âge
}

Patrick Henriet

\section{OpenEdition}

\section{Journals}

Édition électronique

URL : https://journals.openedition.org/ashp/3089

DOI : 10.4000/ashp.3089

ISSN : 1969-6310

Éditeur

Publications de l'École Pratique des Hautes Études

\section{Édition imprimée}

Date de publication : 1 septembre 2019

Pagination : 283-287

ISSN : 0766-0677

\section{Référence électronique}

Patrick Henriet, « Hagiographie, idéologies cléricales et pratiques sociales au Moyen Âge », Annuaire de I'École pratique des hautes études (EPHE), Section des sciences historiques et philologiques [En ligne],

150 | 2019, mis en ligne le 12 juin 2019, consulté le 06 juillet 2021. URL : http:// journals.openedition.org/ashp/3089; DOI : https://doi.org/10.4000/ashp.3089 


\title{
HAGIOGRAPHIE, IDÉOLOGIES CLÉRICALES ET PRATIQUES SOCIALES AU MOYEN ÂGE
}

\author{
Directeur d'études : M. Patrick Henriet
}

Programme de l'année 2017-2018: Pratiques ascétiques et usages du corps dans les textes hagiographiques latins jusqu'au XIII siècle.

Après la lecture et le commentaire de la Vita Romualdi de Pierre Damien (BHL 7324) en 2015-2017, il a été décidé de consacrer au moins deux ans à la question de l'ascétisme et du corps dans les milieux monastiques latins du Moyen Âge. Un exposé général a permis de donner un cadre général aux recherches qui ont été effectuées pendant l'année. Les cas d'ascétisme extrême doivent-ils être interprétés comme des comportements à la limite de la déviance qui situeraient certains saints en marge de l'Église (au sens d'institution)? Cette interprétation a parfois été avancée (cf. par exemple la théorie du pastorat de Michel Foucault, qui a été soumise en séminaire à une critique plutôt serrée), l'ascétisme étant alors saisi dans une grille interprétative qui est aussi celle du mysticisme. Or les nombreux textes convoqués cette année ont montré qu'il fallait plutôt situer cet ascétisme au cœur de l'institution ecclésiale, puisqu'il servit le plus souvent à légitimer ou à réformer l'Église et non à la remettre en cause, sinon dans ses faiblesses et dans ses écarts.

Les comportements ascétiques étant d'une extrême variété, on a choisi de les aborder par le biais de deux pratiques caractéristiques du Moyen Âge central : l'autoflagellation et le port de divers artefacts de fer (chaînes, cercles placés autour du corps, ceintures, cuirasses).

Ont d'abord été étudiés les premiers textes mentionnant des pratiques relevant de la flagellation volontaire (c'est-à-dire non imposée comme punition par une autorité ecclésiastique, en particulier abbatiale). La Vie de saint Pardoux († 737) [BHL 6459], que l'on date du $\mathrm{VIII}^{\mathrm{e}}$ siècle, montre que des pratiques de ce type sont attestées précocement. Il ne s'agit cependant pas encore d'autoflagellation, puisque l'hagiographe montre comment Pardulphe (Pardoux) se faisait fouetter par son disciple Theodenus. Viennent ensuite divers textes hagiographiques la seconde moitié du $\mathrm{XI}^{\mathrm{e}}$ siècle relatifs à Guy de Pomposa († 1046, BHL 8876) et à Poppon de Stavelot († 1048, BHL 6898), dont les vitae sont écrites peu après leur mort, et surtout à Dominique l'encuirassé († 1060), que nous connaissons relativement bien grâce au remarquable récit de Pierre Damien (Ep. 109, éd. Reindel, III, p. 200-223, lettre à Alexandre II donnant les vitae de Raoul de Gubbio et de Dominique). La Vita Dominici (BHL 2239) propose des calculs très précis d'équivalence pénitentielle (nombre de psaumes récités / nombre de coups de verge infligés / nombre d'années de pénitence). Elle a fait l'objet d'une lecture attentive, avec des exercices pratiques destinés à comprendre à quel rythme (élevé) se fouettait le saint ermite. Ce texte constitue un observatoire peut-être unique dans le corpus des sources relatives à l'ascétisme latin du Moyen Âge central, car on y trouve beaucoup d'informations à la fois sur la pratique de l'autoflagellation et sur le port d'une cuirasse pénitentielle, le tout sous la plume d'un auteur exceptionnel. De 
Pierre Damien, on a lu aussi avec une grande attention les passages relatifs à un ascète de la région de Venise qui se fit moine à Fonte Avellana lorsque Pierre en était prieur : ce Michel avait mis au point un dispositif de chaînes très complexe qui lui permettait de tourmenter son corps (Ep. 56, p. 159-160 et Ep. 109, p. 218-219). Ont également été mis en valeur des textes de Pierre Damien qui suggèrent que l'autoflagellation put aussi jouir d'un réel prestige et être pratiquée dans des milieux laïques italiens, dès cette époque et donc bien avant le grand mouvement du XIII ${ }^{\mathrm{e}}$ siècle. Au XII ${ }^{\mathrm{e}}$ siècle, l'autoflagellation était devenue courante chez les ascètes, comme le montre un exemplum du Bonum universale de apibus (II, 16) du dominicain Thomas de Cantimpré $(\dagger 1272)$ : pour avoir refusé cette pratique, Hugues de Saint-Victor est fouetté après sa mort par un grand nombre de démons).

Si l'on ne connaît pas de port de cuirasses pénitentielle en Occident avant l'époque carolingienne, les chaînes et les cercles de fer apparaissent dès le $\mathrm{VI}^{\mathrm{e}}$ siècle. Ils sont déjà bien attestés dans les Vies orientales des Pères du désert (en particulier chez Théodoret de Cyr). Les premières œuvres décrivant leur usage dans le monde latin sont mérovingiennes. Trois textes ont été attentivement lus :

— Grégoire de Tours, Historiae, VI, 6 (éd. Krusch, p. 272-273). Il s'agit sans doute de la première attestation en Occident d'une pratique de cet ordre. Hospitius est reclus à Nice et porte des chaines de fer sous son cilice. Ce détail est d'importance, car il permet dès les origines d'établir un lien entre la future cuirasse métallique et le cilice, celle-là étant en réalité une version exacerbée de celui-ci. Le port de chaînes pénitentielles n'était sans doute pas encore très répandu à l'époque d'Hospitius, puisque les envahisseurs lombards l'interprètent comme le signe qu'Hospitius est un meurtrier. On notera d'ailleurs que cet ascète était peutêtre d'origine orientale : il se nourrit en tout cas d'herbes « égyptiennes » qu'il se procure auprès de marchands (de radicibus herbarum Aegyptiarum, quas eremitae utuntur, exhibentibus sibi negotiatoribus).

- Venance Fortunat, Vie de Radegonde (BHL 7048) (éd. Krusch, MGH, SRM, II, Berlin, 1888, p. 72). Ce passage ne figure pas dans la Vita écrite par Baudonivie mais il a en revanche été retenu dans la réécriture médiévale de BHL 7048 par Hildebert de Lavardin (BHL 7051). Venance raconte comment lors d'un carême, Radegonde avait décidé de porter trois cercles de fer autour du cou et des bras. Trois chaînes étaient reliées aux anneaux. La chair avait très vite recouvert le métal et à la fin du carême, devant l'impossibilité de retirer les cercles, il avait fallu inciser la peau, ce qui avait provoqué d'abondants saignements. La lecture de ce riche texte, qui préfigure par bien des traits des œuvres plus tardives, a l'intérêt de rappeler que le port de chaînes pénitentielles n'était pas un privilège masculin, même s'il est majoritairement attesté pour des hommes. Ce dossier, ici résumé, a également permis de commenter (grâce aux remarques d'une auditrice, Soline Kumaoka) une enluminure du manuscrit 250 de la médiathèque de Poitiers, f. 31v, qui représente Radegonde bardée de chaînes : enluminure particulièrement précieuse dans la mesure où les pratiques dont il est ici question sont très rarement représentées au Moyen Âge.

— Vita A de saint Ouen († 686) [BHL 750]. Dans cette œuvre mérovingienne, il est dit que l'évêque de Rouen avait enserré ses épaules et ses membres dans des 
cercles de fer. Ce choix participait d'un ensemble de pratiques ascétiques plus classiques et faisait de lui l'égal des martyrs qui, "immobilisés par des chaînes de métal, avaient répandu leur précieux sang pour le nom du Christ; de même, ici et en temps de paix, les persécutions étant terminées, il s'était constitué en soldat ardent pour lui-même » (in metallis retrusi pro Christi nomine pretiosum sanguinem fuderunt, ita et hic in pacis tempore, desinente persecutione, ipse sibi fervidus miles institerat...). On retrouve ici le thème bien connu du martyr des ascètes en temps de paix (cf. par exemple Grégoire le Grand).

D'autres textes permettent d'étudier non plus les pénitents volontaires, qui sont généralement de saints hommes ou de saintes femmes sur le modèle d'Hospitius, de Radegonde et de saint Ouen, mais de simples pèlerins, pénitents par la contrainte et condamnés par leur évêque à aller de sanctuaire en sanctuaire jusqu'à la rémission de leurs péchés. Cet aspect du dossier avait jadis été étudié dans un remarquable article du chanoine Platelle, qui avait mis l'accent sur le fait que ces pénitents d'un genre particulier étaient le plus souvent des parricides, le mot devant être entend au sens large (soit tous ceux qui ont tué un parent) ${ }^{1}$. Un récit des miracle de Jean de Réôme rapporte ainsi comment un fratricide chargé de chaînes de fer avait parcouru divers loca sanctorum dont Rome, avant d'être guéri au tombeau du saint (AASS, Janvier, II, I, 3, p. 865, BHL 4430) Le même épisode est mentionné au IX ${ }^{\mathrm{e}}$ siècle dans un ajout opéré au Liber de gloria confessorum de Grégoire de Tours (éd. Krusch, MGH, Script. Rer. Merov., I, p. 353. Cf. Codex Bernensis 199, f. 138). Nous avons attiré l'attention en séminaire sur le commentaire de ce miracle par le jésuite Pierre Rouvier († 1649), dans son édition de l'ensemble du dossier hagiographique de Jean (J. Rouvier, Reomaus seu historia monasterii sancti Ioannis Reomaensis, Paris, 1637).

De fait, sous Charlemagne, un article de l'Admonitio generalis (789) décrit et condamne, sans doute avec peu de succès, " ces gueux et ces vagabonds qui vont sans loi sur cette terre (...). De même pour ces hommes nus et chargés de fer, qui disent errer après avoir reçu une pénitence » (Item ut isti mangones et cotiones, qui sine omni lege vagabundi vadunt per istam terram, [...] nec isti nudi cum ferro, qui dicunt se data sibi poenitentia ire vagantes, éd. H. Mordek et alii, Hanovre, 2012, 77, p. 230). Le dit article est ensuite repris, non sans quelques variantes, au concile de Mayence de 847, puis dans la collection canonique de Réginon de Prüm.

Dans le domaine de la littérature hagiographique, l'un des textes les plus remarquables de cette époque est sans doute un récit des miracles de Florent de Saumur sur lequel on s'est longuement arrêté (BHL 3049, AASS, Sept. VI, col. 431E-432B).

Ce texte posait en effet d'abord un problème de datation qu'il a fallu essayer de résoudre. Le dossier de Florent de Saumur (ou Florent du Mont-Glonne) est en effet complexe et appelle une réédition (Soline Kumaoka, auditrice régulière du séminaire, a entrepris cette tâche). Nous ne dresserons pas ici un état de la question mais nous restreindrons notre analyse, très résumée, au miracle qui nous intéresse.

Ce texte capital explique comment en «Francie intérieure», l'usage s'était répandu de confectionner des liens de fer avec l'épée qui avait tué un parent. Ceint

1. H. Platelle, « Pratiques pénitentielles au Moyen Âge : la pénitence des parricides et l'esprit d'ordalie », dans Mélanges de Science religieuse, 40, 1983, p. 129-155, repris dans Présence de l'au-delà. Une vision médiévale du monde, Villeneuve d'Ascq, 2004. 
de ces liens, le coupable devait ensuite s'exiler et se rendre à Rome et dans d'autres sanctuaires jusqu'à l'obtention du pardon de Dieu. Suit un miracle illustrant cette coutume.

Dans la tradition manuscrite, ce miracle n'est pas intégré à l'ensemble Vita + Miracula mais figure à la fin comme une sorte d'annexe. Il doit donc être daté à part. Il est précédé, point important, d'un court prologue qui semble rédigé sous Charlemagne ou peu après et fait allusion aux progrès de la culture sous le règne de celui-ci. Les bollandistes avaient donc fait de cet appendice un texte carolingien. Cependant, dans un article paru en 1971, Maurice Hamon s'est interrogé sur cette datation et a semblé pencher plutôt, non sans prudence, vers le $\mathrm{X}^{\mathrm{e}}$ ou le $\mathrm{XI}^{\mathrm{e}}$ siècle ${ }^{2}$. Nous ne ferons que résumer ici les arguments qui, au cours d'une longue discussion présentée en séminaire, nous ont clairement fait pencher du côté des bollandistes. Selon Hamon, la datation carolingienne est douteuse car : 1) «l'étrange peine qui frappe le héros du récit et qui évoque de prime abord les lois barbares ne se rencontre en fait dans aucune de ces dernières ». 2) L'hagiographe se plaindrait des leçons de l'office du saint, ce qui pourrait correspondre aux préoccupations d'Ingelbert, auteur d'une Vita de Florent (en grande partie perdue) vers 960-970. 3) L'allusion à la Renaissance carolingienne a pu être faite «bien après les événements en question ». 4) Le texte mentionne un évêque d'Utrecht ou de Maastricht nommé Artcharius. Or reprenant le commentaire des bollandistes, Hamon constate : on n'a « nulle trace au $\mathrm{IX}^{\mathrm{e}}$ siècle d'un Artcharius à Utrecht ou Maastricht, on repère seulement un Hircarius en 840 à Liège ».

Ces quatre arguments ont été discutés, avec les résultats suivants : 1) Comme cela a déjà été dit, l'Admonitio generalis (789) mentionne sous Charlemagne « ces hommes nus et chargés de fer, qui disent errer après avoir reçu une pénitence ». Cette peine est donc bien attestée et n'a rien de surprenant. 2) L'expression series lectionis ne renvoie sans doute pas à l'office comme l'affirme Hamon, mais simplement aux écrits relatifs à Florent. Il s'agit d'un syntagme fréquent et ancien qui, le plus souvent, n'a pas un sens liturgique. 3) L'allusion à Charlemagne semble bien être faite par un auteur qui vit plutôt du vivant de celui-ci ou peu après (liberales siquidem artes usque temporibus domni Karoli imperatoris prcedecessoribus nostris ita extiterunt extranea). Pourquoi l'hagiographe aurait-il menti sur ce point? 4) Vient ici, sans doute, l'argument le plus fort. On ne connait pas toujours bien les dates des anciens évêques d'Utrecht, mais l'argumentation d'Hamon ne tient pas. En effet les bollandistes, dans un autre passage, signalent que le nom Artcharius est sans doute corrompu et ils mentionnent un évêque d'Utrecht appelé Harmackarum ou Hermocarum (il existe d'autres variantes), actif jusqu'en 808-809 ou même 827 selon certains. De fait, il y a bien eu un évêque Harmokarus (autre graphie) à Utrecht entre 791 et v. 804, soit sous Charlemagne ${ }^{3}$. C'est évidemment un élément très fort en faveur d'une datation carolingienne de notre texte : on ne voit guère, si notre hagiographe écrivait au $\mathrm{X}^{\mathrm{e}}$ siècle, où il aurait pris ce nom qu'il a sans doute appris de la bouche $\mathrm{du}$ pèlerin.

2. M. Hamon, «La vie de saint Florent et les origines de l'abbaye du Mont-Glonne », dans Bibliothèque de l'École des chartes, 129, 1971, p. 215-238.

3. Ioannes de Beka, De episcopis ultraiectinis, Utrecht, 1643, p. 43; W. Smith et H. Wace, A Dictionary of Christian Biography, II, Londres, 1880, s.v. « Harmacarus ». 
Les dossiers de pénitents porteurs de chaînes se comptent par dizaines, il n'est donc pas possible de les traiter tous. Nous avons choisi de lire aussi celui de Berthe de Blangy $(† 723)$, un texte qui date peut-être des environs de l'an Mil et qui rapporte la guérison d'un pèlerin porteur de chaînes à l'époque de Charles le Chauve $(B H L$ 1267).

Ces textes ont aussi été lus avec un arrière-plan : faut-il vraiment les dissocier de ceux qui montrent des pénitents volontaires portant des cuirasses métalliques, ainsi que l'a fait Katherine Allen Smith dans un livre qui a été longuement discuté ${ }^{4}$ ? L'œuvre de Pierre Damien suffit à montrer que ce n'est certainement pas le cas. On réserve à l'année 2018-2019 la poursuite de cette étude, qui privilégiera pour commencer deux dossiers qui permettent d'étudier le rapport entre chaînes et cuirasses et auxquels il a simplement été fait allusion. Ces dossiers concernent deux pèlerins allemands, le premier au sanctuaire d'Émeric de Hongrie, l'autre à Malmesbury.

Comme les autres années, la première partie du séminaire a souvent été consacrée à la présentation de travaux plus ou moins récents mais aussi à celle d'œuvres du passé qui permettent de poser sur les textes médiévaux latins un regard parfois inattendu. On citera en particulier une présentation de William Morris (1834-1896), à la suite d'une importante exposition tenue à Madrid, ou encore la lecture d'une lettre de Jacqueline Pascal (sœur Sainte-Euphémie) à son frère Blaise à propos des excès de l'ascétisme (23 juin 1655), Jacqueline s'appuyant dans ses critiques sur l'autorité de saint Bernard. Le directeur d'études a par ailleurs tenu au courant ses auditeurs de l'avancée du livre des 150 ans de l'EPHE, qu'il coordonnait, et il en a extrait plusieurs dossiers qu'il a présentés.

Enfin, une séance entière a été consacrée aux travaux de Julia Smith, professeur à Oxford, qui nous a fait l'honneur d'une conférence sur « Les reliques : une question à repenser par les sciences sociales. Des bénédictions matérielles aux gages spirituels, vers 300 - vers $800 »$ (20 mars 2018).

4. K. Allen Smith, War and the Making of Medieval Monastic Culture, Woodbridge, 2011. 